\title{
Conocimientos y percepciones de los internos de medicina sobre atención primaria de salud con enfoque en salud familiar y comunitaria
}

\section{Knowledge and perceptions of medical interns about primary health care with a focus on family and community health}

\author{
Angielina E. Rosales Anca ${ }^{1, a}$ \\ ${ }^{1}$ Universidad Nacional Mayor de San Marcos. Lima, Perú. \\ ${ }^{a}$ Cirujano Dentista
}

\begin{abstract}
Correspondencia:
Angielina Elizabeth Rosales Anca angielinaelizabeth@gmail.com Dirección: San Martin de Porres 456. Lima, Perú.

Teléfono: 994790084

Recibido: 25 marzo 2018

Aceptado: 3 abril 2018

Conflictos de interés: Ninguno.

Fuentes de financiamiento:

Autofinanciado.

Citar como: Rosales Anca N.

Conocimientos y percepciones de los internos de medicina sobre atención primaria de salud con enfoque en salud familiar y comunitaria. An Fac med. 2018;79(1):98-99

DOl: $h$ ttp://dx.doi.org/10.15381/anales. v79i1.14601
\end{abstract}

\section{An Fac med. 2018;79(1):98-99 / http://dx.doi.org/10.15381/anales.v79i1.14601}

Sr. Editor, el presente estudio tiene como referente los documentos de la Organización Panamericana de la Salud, referidos a la atención primaria de salud (APS) en las américas $(1,2,3)$. Entre las experiencias y aportes de las escuelas de medicina en la APS, Cuba y Brasil son los países de América Latina que han demostrado tener una iniciativa y un alto compromiso gubernamental en el desarrollo de ella, enfatizando la formación de pregrado ${ }^{(3,4,5)}$. Sin embargo, en la mayor parte de las escuelas médicas de las américas, los hospitales continúan siendo el único escenario para el entrenamiento práctico de los estudiantes, dejando de lado el enfoque familiar y comunitario ${ }^{(6)}$.

En la universidad de Cuenca, Ecuador, durante el año 2013 se realizó un estudio de conocimientos y percepciones en APS en una muestra de 143 estudiantes de quinto y sexto año. Como resultado, 54,55\% consideró que existen motivos para justificar el aprendizaje teórico práctico obligatorio ${ }^{(7)}$. En dos universidades de Bogotá, Colombia, el año 2013 un estudio de conocimientos y percepciones sobre APS en estudiantes de décimo semestre de medicina, demostró un desempeño bajo en la evaluación de los conocimientos, asimismo su percepción fue haber recibido información, pero no la suficiente ${ }^{(8)}$. En Lima, Perú, en el año 2006 un estudio en cinco hospitales generales de Lima y Callao, permitió conocer las expectativas acerca del ejercicio profesional e inclinación por la APS en una muestra de 207 internos de medicina; así, el 84,4\% señaló como prioridad, realizar alguna especialidad luego de terminar sus estudios de pregrado y solo el $25,1 \%$ refirieron que se dedicarían a la APS muchos de ellos sin tener un concepto claro acerca de la estrategia ${ }^{(9)}$. En el año 2013 se realizó un estudio en las universidades con mayor demanda de formación en medicina en el Perú, se obtuvo que el $60 \%$ de estas instituciones han incluido contenidos de APS en su currículo; sin embargo, son las escuelas de enfermería y obstetricia donde mejor se ha realizado el cam- 
Tabla 1. Relación entre el nivel de conocimientos y el tipo de percepción de los participantes.

\begin{tabular}{lcccccccc} 
Nivel de & \multicolumn{3}{c}{ Tipo de Percepción } & \multicolumn{2}{c}{ Total } \\
Conocimientos & \multicolumn{2}{c}{ De acuerdo } & \multicolumn{2}{c}{ Indiferente } & \multicolumn{2}{c}{ En desacuerdo } & \multicolumn{2}{c}{} \\
& $\mathbf{n}$ & $\%$ & $\mathbf{n}$ & $\%$ & $\mathbf{n}$ & $\%$ & $\mathbf{n}$ & $\%$ \\
\hline Alto & 1 & $1,9 \%$ & 0 & $0 \%$ & 0 & $0 \%$ & 1 & $0,9 \%$ \\
Medio & 35 & $66 \%$ & 22 & $52,4 \%$ & 2 & $15,4 \%$ & 59 & $54,6 \%$ \\
Bajo & 17 & $32,1 \%$ & 20 & $47,6 \%$ & 11 & $84,6 \%$ & 48 & $44,4 \%$ \\
Total & 53 & $100 \%$ & 42 & $100 \%$ & 13 & $100 \%$ & 108 & $100 \%$ \\
\hline
\end{tabular}

bio del modelo curricular reorientado hacia la APS ${ }^{(10)}$.

Nuestro estudio se realizó en la Facultad de Medicina de la Universidad Nacional Mayor de San Marcos en Lima, Perú. Es una investigación cuantitativa, no experimental, transversal y descriptiva. La población de estudio estuvo conformada por 118 internos de medicina. La selección de la muestra fue de tipo censal, se decidió trabajar con toda la población de estudio. La muestra final quedó reducida a 108 internos de medicina debido a los criterios de inclusión y exclusión. Como técnica se utilizó la encuesta y como instrumento, dos cuestionarios, el primero con preguntas estructuradas autodesarrolladas que permitieron evaluar los conocimientos de los estudiantes y el segundo un cuestionario tipo Likert que permitió valorar las percepciones de los mismos. Los cuestionarios fueron sometidos a los procedimientos de confiabilidad y validación .Para el análisis de confiabilidad, se realizó una prueba piloto a un grupo de 22 estudiantes de una facultad de medicina de otra universidad que presentaron características similares a la población de estudio, los resultados de la prueba piloto se tabularon y se procedió a evaluarlos mediante la validación estadística utilizando el coeficiente alfa de Cronbach. Los valores obtenidos fueron 0,751 en el cuestionario de conocimientos y 0,935 en el cuestionario de percep- ciones, resultando ambos instrumentos confiables. Asimismo, el cuestionario fue validado mediante un juicio de cinco expertos. En la presente investigación se utilizó los paquetes estadísticos Statistical Package for the Social Sciences (SPSS) versión 22, StatKey, y el software Microsoft Excel 2013.

Entre los resultados obtenidos, los participantes presentaron un nivel de conocimiento medio en un $54,6 \%$ y un tipo de percepción "De acuerdo" en un $49,1 \%$ sobre el tema APS con enfoque en salud familiar y comunitario. Asimismo, no se evidencio una asociación significativa entre el nivel de conocimiento y tipo de percepción en APS con enfoque en salud familiar y comunitaria.

Consideramos importante tomar en cuenta la introducción de contenidos académicos en APS y salud familiar y comunitaria de manera longitudinal desde el inicio de la carrera médica, logrando una experiencia temprana en la comunidad que favorecerá el desarrollo de competencias en este ámbito.

\section{REFERENCIAS BIBLIOGRÁFICAS}

1. Organización Panamericana de la Salud. La renovación de la Atención Primaria en Salud en las Américas. Documento de posición de la Organización Panamericana de la Salud y la Organización Mundial de la Salud. Washington D.C.: OPS. 2007. [Fecha de acceso 20 de marzo de 2018]. Disponible en: http://www1.paho.org/hq/ dmdocuments/2010/Renovacion_Atencion_Primaria_Salud_Americas-OPS.pdf

2. Organización Panamericana de la Salud. Serie la renovación de la atención primaria de salud en las américas No.1. Sistemas de salud basados en la Atención Primaria de Salud: Estrategias para el desarrollo de los equipos de APS. Washington D.C.: OPS. 2008. [Fecha de acceso 20 de marzo de 2018]. Disponible en: http://new.paho.org/hq/ dmdocuments/2010/APS-Estrategias_DesarroIlo_Equipos_APS.pdf

3. Organización Panamericana de la Salud. Serie La renovación de la atención primaria de salud en las américas No.2. La Formación en Medicina Orientada hacia la Atención Primaria de Salud. Washington D.C.: OPS. 2008. [Fecha de acceso 20 de marzo de 2018]. Disponible en: http://new.paho. org/hq/dmdocuments/2010/APS-Formacion_Medicina_Orientada_APS.pdf

4. Jardines Méndez J B, Aneiros Riba R, Salas Perea R S. Cuba: Recursos humanos en la atención primaria de salud y su estrategia de desarrollo. Revista Educación Médica y Salud. 1993; 27(2):145-159.

5. Vidal Ledo M, Lemus E R. Docencia de pregrado en Atención Primaria de Salud. Revista Educación Médica Superior. 2012. 26(2):1-11.

6. Fernández Ortega M, Ponce Rosas R, Monobe Hernández C, Landgrave Ibáñez S. Los programas de medicina familiar en las Facultades y Escuelas de Medicina en México. Revista de la Facultad de Medicina. UNAM, 2002. 45 (1):32-4. Disponible en: http://www.revistas.unam.mx/index.php/rfm/ article/view/12646/11966

7. Barros Gavilanes P A, Albán Feijóo G G. Conocimientos y Percepciones de los estudiantes sobre Atención primaria de salud (Tesis de pregrado). Cuenca-Ecuador. Universidad de Cuenca. 2012:122 pp. Disponible en: http://dspace.ucuenca.edu.ec/handle/123456789/3441

8. Manzano M L, Novoa Cely A M Conocimientos y percepciones de estudiantes de medicina de decimo semestre en atención primaria de salud: una mirada general en dos universidades de Bogotá (Tesis de maestria). Bogotá-Colombia. Universidad Nacional de Colombia y la Pontificia Universidad Javeriana. 2013:188 pp. disponible en: https:// repository.javeriana.edu.co/handle/10554/12059

9. Ramirez Huaranga MA. Expectativas de los internos de medicina humana de 5 hospitales generales de Lima y Callao acerca de su ejercicio profesional y su inclinación por la atención primaria de salud (Tesis de pregrado). Lima- Perú. Universidad Nacional Mayor de San Marcos. 2008:60 pp.

10. Ministerio de Salud del Perú. Segunda medición de las metas regionales de los recursos humanos para la salud Perú 2007-2015. Dirección General de Gestión del Desarrollo de Recursos Humano. Serie Bibliográfica Recursos Humanos en Salud N ${ }^{\circ}$ 15. 1ra edición Lima: Sinco Editores, 2013. 